\title{
Perspectivas do manejo terapêutico em pacientes com fibromialgia: uma revisão integrativa
}

\author{
Perspectives of therapeutic management in patients with fibromyalgia: an integrative \\ review
}
Perspectivas del manejo terapéutico en pacientes con fibromialgia: una revisión integradora

Mariana Lima Montenegro ${ }^{1 *}$, Brenda Mendes Veloso², Lara de Vargas Tibério ${ }^{2}$, Larissa Guasti Andrade $^{2}$, Maria Eduarda Almagro Rosi ${ }^{2}$, Matheus Rodrigues Sardinha Drumond Freire ${ }^{2}$, Maurício Polese Juliatti², Nikhole Oliveira², Rhane Pereira da Silva², Thamires Mendes Veloso².

\section{RESUMO}

Objetivo: Descrever o manejo de pacientes com fibromialgia (FM) no que concerne à utilização de terapêuticas combinadas ou alternativas, com a finalidade de delinear quais seriam as condutas mais adequadas a serem adotadas em portadores da síndrome. Métodos: Trata-se de uma revisão bibliográfica do tipo integrativa, de abordagem qualitativa e objetivo descritivo de estudos nacionais e internacionais. Através da base de dados Biblioteca Virtual de Saúde (BVS) e seus sites aliados, foram selecionados e analizados 22 artigos. Resultados: $13,04 \%(n=3)$ dos estudos trazem os exercícios físicos, principalmente aeróbicos, como propulsores de alívio dos sintomas da FM e da melhora na qualidade de vida relacionada à saúde. Ademais, 8,69\% $(n=2)$ dos estudos evidenciaram que exercícios aquáticos são tão eficientes quanto os exercícios terrestres no que tange à melhora do quadro álgico. A neurodinâmica ativa e a estimulação transcraniana do córtex pré-frontal dorsolateral mostraram-se como alternativas para o alívio geral desses pacientes. Considerações finais: Conclui-se que a prática de atividades físicas possui papel preponderante no manejo da FM. Contudo, é evidente a necessidade de estudos mais consistentes para o aperfeiçoamento da terapêutica.

Palavras-chave: Fibromialgia, Controle da dor, Tratamento farmacológico, Terapia combinada, Qualidade de vida.

\section{ABSTRACT}

Objective: To describe the management of patients with fibromyalgia (FM) with regard to the use of combined or alternative therapies, in order to outline what would be the most appropriate approaches to be adopted in patients with the syndrome. Methods: This is an integrative literature review, with a qualitative approach and a descriptive objective of national and international studies. Through the Virtual Health Library (VHL) database and its allied websites, 22 articles were selected and analyzed. Results: 13.04\% $(n=3)$ of the studies bring physical exercises, mainly aerobic, as propellants to relieve FM symptoms and improve health-related quality of life. In addition, $8.69 \%(n=2)$ of the studies showed that aquatic exercises are as efficient as terrestrial exercises in terms of improving pain. Active neurodynamics and transcranial stimulation of the dorsolateral prefrontal cortex proved to be alternatives for the general relief of these patients. Final considerations: It is concluded that the practice of physical activities has a predominant role in the management of FM. However, there is a clear need for more consistent studies to improve therapy.

Keywords: Fibromyalgia, Pain control, Pharmacological treatment, Combination therapy, Quality of life.

1 Universidade Federal do Ceará (UFC), Fortaleza - CE. *E-mail: marilimam20@gmail.com

${ }^{2}$ Centro Universitário do Espírito Santo (UNESC), Colatina - ES.

SUBMETIDO EM: 6/2021

ACEITO EM: 6/2021

PUBLICADO EM: 6/2021 


\section{RESUMEN}

Objetivo: Describir el manejo de los pacientes con fibromialgia (FM) con respecto al uso de terapias combinadas o alternativas, con el fin de delinear cuáles serían los enfoques más adecuados a adoptar en pacientes con el síndrome. Métodos: Se trata de una revisión bibliográfica integradora, con enfoque cualitativo y objetivo descriptivo de estudios nacionales e internacionales. A través de la base de datos de la Biblioteca Virtual en Salud (BVS) y sus sitios web aliados, se seleccionaron y analizaron 22 artículos. Resultados: el 13,04\% $(n=3)$ de los estudios aportan ejercicios físicos, principalmente aeróbicos, como propulsores para aliviar los síntomas de la FM y mejorar la calidad de vida relacionada con la salud. Además, el $8,69 \%(n=2)$ de los estudios mostró que los ejercicios acuáticos son tan eficaces como los terrestres en cuanto a mejorar el dolor. La neurodinámica activa y la estimulación transcraneal de la corteza prefrontal dorsolateral demostraron ser alternativas para el alivio general de estos pacientes. Consideraciones finales: Se concluye que la práctica de actividades físicas tiene un papel predominante en el manejo de la FM. Sin embargo, existe una clara necesidad de estudios más consistentes para mejorar la terapia.

Palabras clave: Fibromialgia, Control del dolor, Tratamiento farmacológico, Terapia combinada, Calidad de vida.

\section{INTRODUÇÃO}

Fibromialgia é uma síndrome clínica crônica que afeta cerca de $2,5 \%$ da população brasileira, com predomínio no sexo feminino e na faixa etária de 35 a 44 anos (HEYMANN RE, et al., 2017). É um distúrbio que gera impacto negativo na qualidade de vida dos pacientes, além de gerar elevados custos econômicos à saúde pública (CABO-MESEGUER A, et al., 2017).

Sabe-se que as hipóteses mais atuais acerca da fisiopatologia da Fibromialgia envolvem mecanismos centrais de modulação e amplificação da dor, o que poderia desencadear tanto uma deficiência de neurotransmissores inibitórios em níveis espinhais ou supraespinhais (serotonina, encefalina, norepinefrina) quanto uma hiperatividade de neurotransmissores excitatórios, como substância $\mathrm{P}$, glutamato e bradicinina (CHINN S, et al., 2016).

Estudos sobre os fenômenos fisiopatológicos que envolvem a fibromialgia também indicam que esta condição pode ser considerada uma síndrome de sensibilidade central, ou seja, que apresenta relação com o processamento anormal da dor. Com isso, tem crescido o número de estudos que demonstram evidências significativas sobre a presença de mecanismos fisiopatológicos inflamatórios envolvidos nesta síndrome de dor crônica, que afeta cérebro, medula espinhal e tecidos periféricos por meio da ativação do sistema imunológico inato e adaptativo, através da ativação de citocinas, quimiocinas, estresse oxidativo e neuropeptídeos. Em consonância, também é sabido que o estado emocional e o estresse psicológico podem estimular a inflamação neurogênica, contribuindo ainda mais para a sintomatologia do quadro álgico (LITTLEJOHN G e GUYMER E, 2018; BENLIDAYI CI, 2019).

Certamente, a hereditariedade é um componente para o desenvolvimento da fibromialgia. Foi demonstrado que parentes de primeiro grau possuem oito vezes mais risco de desenvolver a síndrome quando comparados ao resto da população. Nesta perspectiva, estudos gênicos investigaram o possível envolvimento entre a patogenia da fibromialgia e fatores genéticos, que são potencialmente responsáveis por $50 \%$ da susceptibilidade à síndrome (D'AGNELLI S, et al., 2019).

Segundo de Lorena SB, et al. (2016) os possíveis sintomas que constituem o quadro clínico da fibromialgia podem ser físicos ou mentais, destacando-se fadiga generalizada, distúrbios do sono, rigidez matinal, sensação de dispneia, ansiedade, depressão e alteração da função cognitiva. Todas estas apresentações são correlacionadas com a dor, o que constitui um sinal de alerta para a busca do tratamento por ocasionar limitação da realização das atividades de vida diária e profissional.

Por se tratar de uma doença com influência de aspectos sociais, psicológicos e culturais, sua expressão clínica é altamente variável e, portanto, de difícil diagnóstico. Além disso, a ausência de marcadores clínicos ou laboratoriais objetivos dificulta o seu rastreio (MARTINEZ JE, 2017). Nesta perspectiva, Heymann RE, et 
al. (2017) formularam as novas diretrizes para o diagnóstico da fibromialgia, com atualizações das versões anteriores de 1990 e 2010, que eram enviesadas por supervalorizar a dor difusa em detrimento de outros sintomas, como rigidez matinal, alterações do sono e fadiga.

No que tange à valorização de outros sintomas para definição diagnóstica da fibromialgia, observou-se que a classificação de pontos dolorosos não tem sido adequadamente utilizada por profissionais não treinados e, portanto, está associada a subnotificação, por desvalorizar sinais e sintomas importantes, como ansiedade, depressão, cansaço matinal, distúrbios do sono e problemas cognitivos. A nova diretriz diagnóstica recomenda avaliar outros fatores de risco, como artrite reumatoide, depressão e síndrome do pânico, todos necessariamente aliados a uma correlação clínica que possa sugerir o diagnóstico. Ademais, vale salientar que a diretriz mais recente não considera válida a realização de termografia e polissonografia, pela falta de evidências científicas que corroborem com sua solicitação (HEYMANN RE, et al., 2017).

Segundo Sarzi-Puttini P, et al. (2020) o manejo terapêutico da FM deve ser iniciado com abordagens integradas e multidisciplinares, já vista ser uma patologia caracterizada por uma conexão mente-corpo. $O$ condicionamento físico, o tratamento farmacológico, a psicoterapia e por fim a educação do paciente devem estar associados para se obter a melhora do quadro clínico. Para iniciar o tratamento a terapia farmacológica de imediato é indicada, uma vez que, normalmente esses pacientes são diagnosticados anos após o inicio dos sintomas, porém, de fato esses pacientes só melhoram substancialmente com a adoção de outros métodos terapêuticos como o tratamento não farmacológico, além de estratégias básicas de autocuidado. Portanto, vale ressaltar que o tratamento individualizado e centrado no paciente é de suma importância.

A farmacoterapia objetiva a analgesia orientada de acordo com o mecanismo de ação de cada medicamento, entretanto junto a eles está associado a possibilidade de efeitos adversos. Já a terapia alternativa, não farmacológica, sugere ter maior magnitude de efeitos multidimensionais em relação ao tratamento farmacológico no manejo terapêutico da FM, porém esta terapia ainda possui certas controvérsias, assim carecendo de estudos com qualidades de evidências mais fortes (SARZI-PUTTINI P, et al., 2020).

Diante deste contexto, emerge o seguinte questionamento: que estratégias terapêuticas seriam ideais na abordagem de pacientes com fibromialgia? Esta pesquisa tem por objetivo descrever o manejo de pacientes com fibromialgia no que concerne à utilização de terapêuticas combinadas ou alternativas, com o fito de delinear quais seriam as condutas mais adequadas a serem adotadas em portadores da síndrome.

\section{MÉTODOS}

Trata-se de uma revisão bibliográfica do tipo integrativa, com abordagem qualitativa, de procedimento bibliográfico, natureza aplicada e objetivo descritivo de estudos nacionais e internacionais. Neste sentido, através da utilização de Descritores em Ciências da Saúde nos idiomas português, inglês e espanhol, foi configurada a seguinte fórmula de busca que, em sequência, seria utilizada no banco de dados Biblioteca Virtual de Saúde: "(Síndrome de Fadiga Crônica OR Fatigue Syndrome OR Síndrome de Fatiga Crónica OR Syndrome de fatigue chronique OR Fibromialgia OR Fibromyalgia OR Fibromyalgie OR Síndrome da Dor Miofascial Difusa) AND (Terapêutica OR Ação Terapêutica OR Ações Terapêuticas OR Medida Terapêutica OR Medidas Terapêuticas OR Procedimento Curativo OR Procedimento de Terapia OR Procedimento de Tratamento OR Procedimento Terapêutico OR Procedimentos Curativos OR Procedimentos de Terapia OR Procedimentos de Tratamento OR Procedimentos Terapêuticos OR Propriedade Terapêutica OR Terapia OR Terapias OR Tratamento OR Tratamentos OR Therapeutics OR Therapy OR Treatment OR Terapéutica OR Thérapeutique)".

Por conseguinte, de acordo com os resultados obtidos a partir da fórmula de busca supracitada, foram encontrados 40 artigos no total, os quais sofreram um segundo processo de triagem, tendo sido excluídos aqueles publicados antes de 2015 , bem como os trabalhos de metanálise e revisão sistemática. Nesta perspectiva, foram incluídos artigos que atendessem aos seguintes critérios: publicação nos últimos cinco anos; ensaios clínicos controlados; artigos em português, inglês, espanhol ou francês; artigos que respondem à questão de pesquisa. Finalmente, restaram 22 artigos, os quais compõem esta revisão de literatura e são analisados no que tange aos seus principais desfechos (Figura 1). 
Figura 1 - Fluxograma de identificação e seleção de artigos.

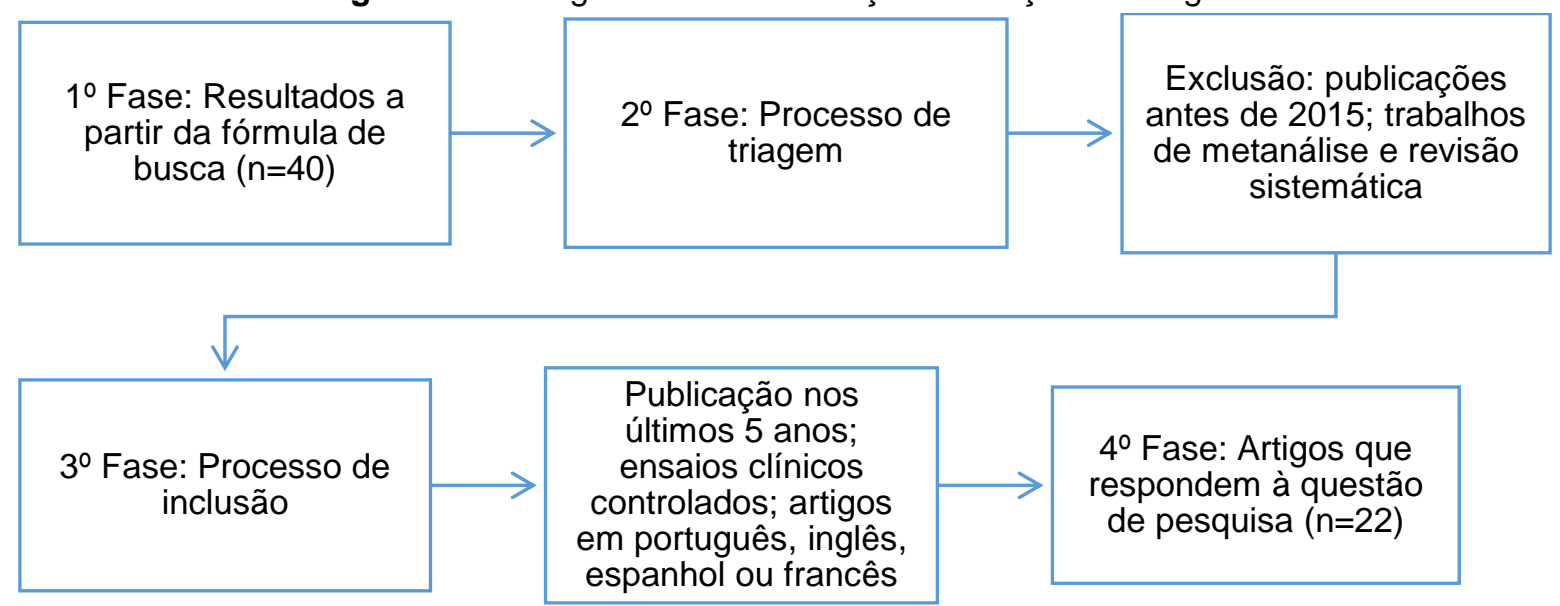

Fonte: Montenegro ML, et al., 2021.

\section{RESULTADOS}

O Quadro 1 descreve uma síntese geral dos vinte e dois artigos selecionados para a efetivação deste estudo. Nos quais são mostradas diferentes perspectivas relacionadas ao manejo terapêutico de pacientes com fibromialgia.

Dentre os artigos selecionados, $13,04 \%(n=3)$ analisaram a prática de exercícios físicos, principalmente aeróbicos, como mediadores de melhoras na qualidade de vida dos pacientes e alívio de sintomas da fibromialgia, dentre os quais fadiga e dor. Estudo de Kim SY, et al. (2019) comparou o treinamento aeróbico com o treinamento de flexibilidade, tendo sido demonstrada ineficácia, a curto ou longo prazo, na diminuição da intensidade das dores e da fadiga.

Em contrapartida, 8,69\% $(n=2)$ dos estudos evidenciaram benefícios importantes atrelados à prática de exercícios aquáticos, tanto como redutores dos sintomas quanto como propulsores de melhora no condicionamento aeróbico e físico. Tais atividades foram identificadas como tão eficientes quanto os exercícios terrestres, exceto no quesito força muscular, que se mostrou ser mais adequadamente estimulado nas práticas terrestres (RIVAS NEIRA S, et al., 2017).

De uma forma geral, portanto, as recomendações para alívio de dor em pacientes com fibromialgia envolvem a prática de exercícios físicos. As evidências, apoiadas em ensaios clínicos randomizados, demonstram vantagens importantes relacionadas à realização de exercícios aeróbicos, associação de exercícios mistos, prática de pilates em conjunto a exercícios aquáticos e treinamentos de auto-liberação miofascial (BIDONDE J, et al., 2017; BIDONDE J, et al., 2019; SILVA HJDA, et al., 2019; CECA D, et al., 2017).

Para Fitzgibbon BM, et al. (2018), To WT, et al. (2017) e Torres JR, et al. (2015) a neurodinâmica ativa funciona como atenuante das dores e da fadiga. Neste contexto, a estimulação do córtex pré-frontal dorsolateral, em um tratamento diário de 4 semanas, foi correlacionada com diversos benefícios em relação aos sintomas álgicos da fibromialgia. Além disso, vale salientar que sessões repetidas de estimulação de uma corrente contínua transcraniana do córtex pré-frontal dorsolateral evidenciou, também, redução da dor em pacientes com esta síndrome.

Em relação ao desenvolvimento da fibromialgia, sabe-se que a disfunção do sono pode ser uma das causas precipitantes. Observou-se que distúrbios do ciclo sono-vigília afetam o processamento da dor e, dessa forma, reduzem a sua modulação descendente. Esta informação atesta, portanto, que a boa qualidade do sono tem implicação direta na melhora da sintomatologia característica da síndrome da dor miofascial difusa. Nesta perspectiva, algumas das estratégias de enfrentamento da fibromialgia envolvem a melhoria da qualidade do sono, tendo sido identificado um bom perfil de aceitação com o uso de ciclobenzaprina e de agonistas do receptor de melatonina. Tais medicações mostraram-se promissoras na melhoria do sono e, consequentemente, nos sintomas da fibromialgia ( $\mathrm{CHOY} \mathrm{EH,} \mathrm{2016).}$ 
Quadro 1 - Principais Resultados dos Artigos Selecionados para Pesquisa

\begin{tabular}{|c|c|c|}
\hline Autor/Ano/País & Título & Principais Resultados \\
\hline $\begin{array}{l}\text { Kim SY, et al. (2019), } \\
\text { Estados Unidos }\end{array}$ & $\begin{array}{l}\text { Treinamento de exercícios de flexibilidade para adultos com } \\
\text { fibromialgia. }\end{array}$ & $\begin{array}{l}\text { Exercícios de flexibilidade, quando em comparação com exercícios de resistência e } \\
\text { aeróbicos terrestres, não apresentaram evidência de melhora na fadiga, capacidade } \\
\text { física e sensação de dor nos portadores de fibromialgia. }\end{array}$ \\
\hline $\begin{array}{l}\text { Bidonde J, et al. } \\
\text { (2019), Estados } \\
\text { Unidos }\end{array}$ & Treinamento de exercício misto para adultos com fibromialgia & $\begin{array}{l}\text { Os efeitos do exercício se apresentaram como clinicamente irrelevantes e potenciais } \\
\text { causadores de dor e incômodo no momento da realização do exercício ou após o seu } \\
\text { término. }\end{array}$ \\
\hline $\begin{array}{l}\text { Silva HJDA, et al. } \\
\text { (2019), Brasil }\end{array}$ & $\begin{array}{l}\text { Mat Pilates e exercícios aeróbicos aquáticos para mulheres com } \\
\text { fibromialgia: um protocolo para um estudo cego controlado } \\
\text { randomizado }\end{array}$ & $\begin{array}{l}\text { A prática de exercícios aquáticos como o pilates demonstrou ser tão benéfica para o } \\
\text { tratamento de fibromialgia quanto os exercícios aeróbicos não aquáticos. Ademais, o } \\
\text { treino no ambiente aquático possui evidências da melhoria do bem-estar e da capacidade } \\
\text { física dos pacientes portadores da síndrome. }\end{array}$ \\
\hline $\begin{array}{l}\text { Uysal SC, et al. } \\
\text { (2019), Turquia }\end{array}$ & $\begin{array}{l}\text { Eficácia da técnica de energia muscular na força e resistência } \\
\text { muscular respiratória em pacientes com fibromialgia. }\end{array}$ & $\begin{array}{l}\text { A aplicação da técnica de energia muscular (MET) na musculatura respiratória acessória } \\
\text { cervical dos indivíduos com fibromialgia apresentou a capacidade de fortalecer e } \\
\text { aumentar a resistência da musculatura respiratória, aumentar a flexibilidade cervical, } \\
\text { reduzir o nível de fadiga e dor. }\end{array}$ \\
\hline $\begin{array}{l}\text { Fitzgibbon BM, et al. } \\
\text { (2018), Austrália }\end{array}$ & $\begin{array}{l}\text { Evidências para a melhora da fadiga na fibromialgia: Um estudo } \\
\text { randomizado controlado com estimulação magnética transcraniana } \\
\text { repetitiva do córtex pré-frontal dorsolateral esquerdo de } 4 \text { semanas. }\end{array}$ & $\begin{array}{l}\text { O tratamento diário de } 4 \text { semanas de estimulação magnética transcraniana no córtex pré- } \\
\text { frontal dorsolateral em pacientes com fibromialgia reduz a fadiga e possui maior } \\
\text { probabilidade de reduzir a sensação de dor nesses indivíduos. }\end{array}$ \\
\hline $\begin{array}{l}\text { Hermans L, et al. } \\
\text { (2018), Bélgica }\end{array}$ & $\begin{array}{l}\text { Influência da morfina e da naloxona na modulação da dor na artrite } \\
\text { reumatóide, síndrome da fadiga crônica/ fibromialgia e controles: um } \\
\text { estudo duplo-cego, randomizado, controlado por placebo e cruzado. }\end{array}$ & $\begin{array}{l}\text { A utilização da morfina em pacientes com artrite reumatóide ou fibromialgia apresenta } \\
\text { um efeito limitado. Além disto, o uso da naloxona e da morfina não altera a sensação de } \\
\text { dor no tecido profundo. Tais resultados demonstraram que os opioides não têm influência } \\
\text { na modulação da dor. }\end{array}$ \\
\hline $\begin{array}{l}\text { Bidonde J, et al. } \\
\text { (2017), Estados } \\
\text { Unidos }\end{array}$ & $\begin{array}{l}\text { Treinamento de exercício de vibração de corpo inteiro para } \\
\text { fibromialgia. }\end{array}$ & $\begin{array}{l}\text { A vibração do corpo inteiro em adultos com fibromialgia possui evidências de qualidade } \\
\text { baixa para confirmar ou negar sua eficácia sozinha ou em associação com outros } \\
\text { exercícios para melhorar a qualidade de vida, fadiga, dor e equilíbrio. }\end{array}$ \\
\hline $\begin{array}{l}\text { Bidonde J, et al. } \\
\text { (2017), Estados } \\
\text { Unidos }\end{array}$ & Treinamento de exercícios aeróbicos para adultos com fibromialgia. & $\begin{array}{l}\text { A prática do exercício físico aeróbico em indivíduos com fibromialgia se apresentou } \\
\text { positiva para melhora da dor e qualidade de vida relacionada à saúde, além de um } \\
\text { pequeno aumento na função física e cardiorrespiratória. }\end{array}$ \\
\hline $\begin{array}{l}\text { To WT, et al. (2017), } \\
\text { Estados Unidos }\end{array}$ & $\begin{array}{l}\text { Efeitos diferenciais da estimulação dos nervos bifrontal e occipital na } \\
\text { dor e fadiga usando estimulação transcraniana por corrente contínua } \\
\text { em pacientes com fibromialgia. }\end{array}$ & $\begin{array}{l}\text { A realização de técnicas que estimulam o cérebro, tais como sessões de estimulação } \\
\text { transcraniana por corrente contínua no nervo occipital ou córtex pré-frontal dorsolateral } \\
\text { demonstrou ser eficaz na melhora da dor e fadiga em portadores de fibromialgia. }\end{array}$ \\
\hline $\begin{array}{l}\text { Ceca D, et al. (2017), } \\
\text { Espanha }\end{array}$ & $\begin{array}{l}\text { Benefícios de um programa de liberação auto-miofascial na } \\
\text { qualidade de vida relacionada à saúde em pessoas com fibromialgia: } \\
\text { um ensaio clínico randomizado. }\end{array}$ & $\begin{array}{l}\text { A realização de exercícios de liberação auto-miofascial tem a capacidade de melhorar a } \\
\text { dor e qualidade de vida relacionada à saúde em pessoas que possuem a fibromialgia. }\end{array}$ \\
\hline $\begin{array}{l}\text { Rivas Neira S, et al. } \\
\text { (2017), Espanha }\end{array}$ & $\begin{array}{l}\text { Eficácia da terapia aquática vs terapia terrestre para equilíbrio e dor } \\
\text { em mulheres com fibromialgia: um protocolo de estudo para um } \\
\text { ensaio clínico randomizado. }\end{array}$ & $\begin{array}{l}\text { Ainda são necessários mais estudos para incluir aos protocolos da fisioterapia o } \\
\text { tratamento da fibromialgia. }\end{array}$ \\
\hline
\end{tabular}




\begin{tabular}{|c|c|c|}
\hline Autor/Ano/País & Título & Principais Resultados \\
\hline $\begin{array}{l}\text { Di Pierro F et al. } \\
\text { (2017), Itália }\end{array}$ & $\begin{array}{l}\text { Papel da forma solúvel em água de CoQ10 em mulheres afetadas } \\
\text { pela fibromialgia: um estudo preliminar. }\end{array}$ & $\begin{array}{l}\text { Mulheres portadoras de fibromialgia que receberam uma forma de água solúvel de } \\
\text { CoQ10 apresentaram melhora na sensação de dor e fadiga, além de apresentarem } \\
\text { melhora no sono e em distúrbios mentais, como ansiedade e depressão. }\end{array}$ \\
\hline $\begin{array}{l}\text { Kallese } \mathrm{KH} \text { et al. } \\
\text { (2016), Dinamarca }\end{array}$ & $\begin{array}{l}\text { Comparando aceitação baseada em grupo e terapia de compromisso } \\
\text { (ACT) com o atendimento usual aprimorado para adolescentes com } \\
\text { síndromes somáticas funcionais: um protocolo de estudo para um } \\
\text { ensaio randomizado. }\end{array}$ & $\begin{array}{l}\text { Adolescentes portadores de fibromialgia, síndrome da fadiga crônica e síndromes de dor } \\
\text { idiopática apresentam redução na sua qualidade da vida de maneira geral, além de outras } \\
\text { problemática, tais como: ausência de escolaridade e dificuldades na socialização. }\end{array}$ \\
\hline $\begin{array}{l}\text { Maddali Bongi S, et al. } \\
\qquad(2016) \text {, Itália }\end{array}$ & $\begin{array}{l}\text { Eficácia da reabilitação com Tai Ji Quan em uma coorte italiana de } \\
\text { pacientes com Síndrome de Fibromialgia. }\end{array}$ & $\begin{array}{l}\text { A utilização de terapias corpo-mente como o Tai Ji Quan demonstrou eficácia na redução } \\
\text { de dor, fadiga e incapacidade, além da melhora da qualidade de vida relacionada à } \\
\text { saúde e distúrbios mentais, como depressão e ansiedade. }\end{array}$ \\
\hline $\begin{array}{l}\text { Ericsson A, et al. } \\
\text { (2016), Suécia }\end{array}$ & $\begin{array}{l}\text { O exercício de resistência melhora a fadiga física em mulheres com } \\
\text { fibromialgia: um ensaio clínico randomizado. }\end{array}$ & $\begin{array}{l}\text { A promoção de uma série de exercícios de resistência em mulheres com fibromialgia } \\
\text { demonstrou bons resultados na melhora da fadiga geral, mental e física. }\end{array}$ \\
\hline $\begin{array}{l}\text { Choy EH (2016), } \\
\text { Reino Unido }\end{array}$ & $\begin{array}{l}\text { Tratamentos atuais para combater a disfunção do sono como um } \\
\text { estímulo patogênico da fibromialgia. }\end{array}$ & $\begin{array}{l}\text { Fármacos como Melatonina, inibidores da recaptação de Serotonina, Oxibato de sódio e } \\
\text { Pregabalina demonstram a capacidade de melhorar o sono de pacientes com } \\
\text { Fibromialgia. Ademais, a Melatonina possui a capacidade de reduzir a dor nesses } \\
\text { indivíduos, pois aumentou o sistema inibidor de modulação da dor. }\end{array}$ \\
\hline $\begin{array}{l}\text { Saral I, et al. (2016), } \\
\text { Turquia }\end{array}$ & $\begin{array}{l}\text { Os efeitos das abordagens de tratamento interdisciplinar de curto e } \\
\text { longo prazo em mulheres com fibromialgia: um ensaio clínico } \\
\text { randomizado. }\end{array}$ & $\begin{array}{l}\text { As abordagens de tratamento interdisciplinar de curto e longo prazo na terapia de } \\
\text { mulheres com fibromialgia são eficientes para redução da dor e fadiga. Ademais, as } \\
\text { abordagens também se mostraram importantes na melhora da qualidade de vida } \\
\text { relacionada à saúde e aumento do limiar da dor. }\end{array}$ \\
\hline $\begin{array}{l}\text { Torres JR, et al. } \\
\text { (2015), Espanha }\end{array}$ & $\begin{array}{l}\text { Resultados de um Programa de Mobilização Neurodinâmica Ativa } \\
\text { em Pacientes com Síndrome de Fibromialgia: Um Ensaio Clínico } \\
\text { Randomizado. }\end{array}$ & $\begin{array}{l}\text { Os resultados de um programa de mobilização neurodinâmica demonstrou eficiência na } \\
\text { melhora da dor, fadiga e estado funcional em pacientes diagnosticados com fibromialgia. }\end{array}$ \\
\hline $\begin{array}{l}\text { White HD, et al. } \\
\text { (2015), Estados } \\
\text { Unidos }\end{array}$ & $\begin{array}{l}\text { Tratamento da dor em pacientes com fibromialgia com gel de } \\
\text { testosterona: Farmacocinética e resposta clínica. }\end{array}$ & $\begin{array}{l}\text { Mesmo apresentando resultados que demonstram redução da dor e fadiga em pacientes } \\
\text { com fibromialgia, a utilização da testosterona nesses indivíduos necessita ainda de } \\
\text { estudos adicionais para ser acrescentada na terapia da doença. }\end{array}$ \\
\hline $\begin{array}{l}\text { Regland B, et al. } \\
\text { (2015), Suécia }\end{array}$ & $\begin{array}{l}\text { Resposta à vitamina B12 e ácido fólico na encefalomielite miálgica e } \\
\text { fibromialgia. }\end{array}$ & $\begin{array}{l}\text { O uso de vitamina B12 em combinação ao ácido fólico pode fornecer concentrações } \\
\text { sanguíneas para promover alívio em pacientes com fibromialgia e encefalomielite álgica. }\end{array}$ \\
\hline $\begin{array}{l}\text { Meeus M, et } \\
\text { al. (2015), Bélgica }\end{array}$ & $\begin{array}{l}\text { Modulação endógena da dor em resposta ao exercício em pacientes } \\
\text { com artrite reumatoide, pacientes com síndrome da fadiga crônica e } \\
\text { fibromialgia comórbida e controles saudáveis: um ensaio clínico } \\
\text { duplo-cego randomizado. }\end{array}$ & $\begin{array}{l}\text { Para os pacientes com fibromialgia e síndrome da fadiga crônica os resultados se } \\
\text { apresentaram menos óbvios e, com isso, são necessárias mais pesquisas para } \\
\text { apresentar conclusões mais consistentes sobre o tema. }\end{array}$ \\
\hline $\begin{array}{l}\text { Noehren B, et al. } \\
\text { (2015), Estados } \\
\text { Unidos }\end{array}$ & $\begin{array}{l}\text { Efeito da estimulação elétrica nervosa transcutânea na dor, função e } \\
\text { qualidade de vida na fibromialgia: um ensaio clínico randomizado } \\
\text { duplo-cego. }\end{array}$ & $\begin{array}{l}\text { O aumento no tempo da prática de atividade física diária em pacientes com fibromialgia } \\
\text { é capaz de diminuir a dor, fadiga e promover melhorias na qualidade de vida. }\end{array}$ \\
\hline
\end{tabular}




\section{DISCUSSÃO}

\section{Fisiopatologia}

No que tange aos mecanismos fisiopatológicos atrelados à Fibromialgia, verificou-se que um dos caminhos do trato descendente inibitório do sistema nervoso central tem origem na região rostral vasomedial da medula e no núcleo da rafe e contém neurônios serotoninérgicos e gabaérgicos, que fazem sinapse na medula espinal para inibir a transmissão do impulso doloroso no corno dorsal. Por sua vez, uma segunda via se origina do tegumento dorsolateral posterior, que contém neurônios noradrenérgicos inibitórios (CHINN S, et al., 2016).

Segundo Choy EH (2016), alguns estudos atestaram anormalidades neste trato ao identificar níveis reduzidos de serotonina e norepinefrina no fluido cerebroespinal, bem como atividade reduzida do córtex cingulado anterior, região do cérebro responsável pelas vias inibitórias descendentes da dor. Tais vias abrigam altos níveis de $\mu$-opióides, mas, em pacientes com fibromialgia, estes receptores apresentam diminuição do potencial de ligação aos opioides. Ademais, também foi elencada uma diminuição da sensibilidade aos opioides nestes pacientes, o que corrobora para evidências de ineficácia do tratamento de fibromialgia com esses medicamentos.

Uma outra via envolve o subnúcleo reticular dorsal da região caudal da medula. Este modulador inibitório da dor é responsável pelo "condicionamento nocivo heterópico", fenômeno através do qual a dor de uma parte do corpo reduz os estímulos nocivos da outra região do corpo, também presente em pacientes com síndrome da dor miofascial difusa. (SARZI-PUTTINI P, et al., 2020).

De acordo com Sarzi-Puttini P, et al. (2020), estudos atuais apontam evidências de aberrações em nervos periféricos em pacientes com fibromialgia e outras síndromes crônicas dolorosas. Hipóteses mais recentes sugerem que mediadores de imunidade inata e adaptativa, incluindo mastócitos, células dendríticas e linfócitos $T$, são ativados e geram excitabilidade de vias nociceptivas como resultado de um processo de neuroinflamação. Esta prerrogativa foi postulada com base na detecção de substâncias pró-inflamatórias e autoanticorpos órgãos-específicos e não específicos no soro de pacientes portadores de fibromialgia. Tal neuroinflamação pode ser classificada como clássica, quando envolve um mecanismo de estímulos nociceptivos, ou neurogênica, quando o estresse ou outro fator fisiológico induz a resposta neural a amplificar a percepção da dor.

Como resultado, a presença de certos sinais e achados nos exames de pacientes com fibromialgia corroboram para a percepção deste mecanismo, dentre os quais pode-se citar edema, descoloração reticular da pele, dermatografismo, disestesia cutânea e, até mesmo, fenômeno de Raynaud (SARZI-PUTTINI P, et al., 2020).

Em congruência com Sarzi-Puttini P, et al. (2020), Häuser W, et al. (2015) afirma que a fibromialgia também está associada a uma variedade de estressores psicológicos, incluindo traumas de infância e abusos, estresses diários, exposição à guerra e a catástrofes naturais. A partir destes estressores, extensas pesquisas foram realizadas e revelaram alterações no eixo hipotálamo-pituitária-adrenal e no sistema nervoso simpático em pacientes com fibromialgia que foram vítimas dessas condições.

\section{Manejo}

Muitos fatores contribuem para o desenvolvimento da fibromialgia, dentre os quais pode-se citar predisposição genética, experiências pessoais, fatores emocionais e cognitivos, relação entre corpo e mente e a habilidade de lidar com estressores. Neste sentido, a fibromialgia representa uma conexão entre corpo e mente, o que, consequentemente, expressa a necessidade de um tratamento holístico de caráter multidisciplinar. Häuser W, et al. (2015), assim como Sarzi-Puttini P, et al. (2020) e a Liga Europeia Contra o Reumatismo, propõem um tratamento dividido em 4 pilares: educação do paciente, exercícios físicos, tratamento farmacológico e psicoterapia.

Uma parte importante em lidar com pacientes com fibromialgia é assegurar que o paciente entenda a sua condição antes da prescrição de qualquer medicamento. É de extrema importância comunicar ao paciente 
que fibromialgia é uma patologia real e legitimar o seu sofrimento, além de deixar claro que, mesmo sendo incapacitante, a condição não é progressiva e não causa danos a tecidos periféricos. Os pacientes portadores de tal síndrome devem ser informados sobre seu papel primordial no manejo da fibromialgia e devem desenvolver os seus próprios métodos de melhorar a qualidade de vida. Esse tipo de abordagem é conhecida como "self-management" e apoia-se na ideia de que o paciente é o protagonista do seu tratamento (SARZIPUTTINI P, et al., 2020).

Segundo Bidonde J, et al. (2017), a realização de atividade física aeróbica é uma ferramenta importante de condução dos pacientes com fibromialgia, tendo sido demonstrada melhora da dor, evolução relacionada à qualidade de vida e aumento na função cardiorrespiratória. Da mesma forma, Sarzi-Puttini $P$, et al. (2020) preconizam a adoção do tratamento não farmacológico, pautado em uma vida mais saudável, com mudanças nos hábitos alimentares, diminuição dos quadros de obesidade e introdução da prática de exercícios físicos de fortalecimento.

No entanto, apesar da forte recomendação da prática de exercícios aeróbicos, pode haver certa limitação desta prática em pacientes portadores da síndrome, graças à falta de condicionamento e à presença de fatores fisiológicos restritivos (SARZI-PUTTINI P, et al., 2020). Nesta perspectiva, Bidonde J, et al. (2017) afirmam que a prática de exercícios não se demonstra significativamente benéfica aos pacientes com fibromialgia, podendo ser nociva ao intensificar a dor e provocar desconforto durante ou após a realização das atividades. Em contrapartida, Assumpção A, et al. (2018) evidenciam que a prática de alongamento e resistência muscular realizada por indivíduos com o quadro clínico em questão promove melhora na função física, diminuição da dor e redução da depressão.

Portanto, treinamentos de alongamento muscular são responsáveis por melhorar a qualidade de vida do paciente, sobretudo no que tange aos transtornos funcionais. Ademais, os exercícios de resistência são bastante eficazes para quadros de depressão em pacientes com fibromialgia.

Uma opção citada por Häuser W, et al. (2015) e confirmada pelo estudo de Rivas Neira S, et al. (2017) é a terapia aquática, em virtude das propriedades da água que facilitam a prática de exercícios, reduzem o impacto em articulações, melhoram a microcirculação, facilitam o relaxamento e melhoram o tônus muscular devido a sua resistência natural.

Conforme Kallese KH, et al. (2016), adolescentes que apresentam síndromes somáticas funcionais podem sofrer com a perpetuação de problemas físicos no estado adulto de seu desenvolvimento, portando queixas quanto à incapacidade no trabalho, distanciamento social e transtorno físicos. Dessa forma, nota-se a redução na qualidade de vida como uma das implicações da fibromialgia. Ademais, de La Vega R, et al. (2016) abordam que a síndrome de fibromialgia juvenil também demonstra uma problemática de acessibilidade ao tratamento e apoio, visto que uma pequena parcela de profissionais da saúde está apta e devidamente treinada para auxiliar tais pacientes e suas famílias.

Destaca-se, ainda, outra proposta terapêutica denominada Saúde Móvel, que é aplicada a dispositivos móveis. Essa medida promove economia de tempo e custos, além de proporcionar autonomia e acessibilidade ao tratamento da fibromialgia na juventude. Desse modo, foi desenvolvido um aplicativo para smartphone chamado Fibroline. A ferramenta em questão é capaz de registrar, basicamente, práticas de ioga, sono de qualidade, controle da ansiedade, medicações e condição física do paciente. Estas intervenções via internet evidenciaram eficiência na diminuição da dor e dos transtornos físicos e mentais dos usuários (DE LA VEGA R, et al., 2016).

\section{Farmacoterapia}

Além das terapias não farmacológicas supracitadas ainda existe a farmacoterapia. Essa terapêutica para os pacientes que sofrem da fibromialgia é indicada para alívio dos sintomas, porém somente na minoria dos pacientes é revelado resultados benéficos na utilização destes. Nesses pacientes tratados com medicamentos foi evidenciado uma queda de $50 \%$ da intensidade dor, valor significante para a melhora da qualidade de vida (MAFFEI ME, 2020). 
Vale ressaltar que os medicamentos usados na terapia farmacológica apresentam certas limitações como os efeitos adversos ou eficácia incompleta, por isso atualmente existem hipóteses em que se deve combinar analgésicos com mecanismos de ação diferentes a fim de potencializar sua eficiência e garantir melhores resultados terapêuticos (MAFFEI ME, 2020).

\section{Canabinoides}

Segundo Prabhakar A, et al. (2019) e Chinn S, et al. (2016), estudos com canabinoides demonstraram melhoras na dor, entretanto estão associadas a efeitos adversos muito leves, consistindo de boca seca, olhos vermelhos e sensação de fome. Alguns pacientes, os quais utilizam esta forma terapêutica, deixaram de usar qualquer outro medicamento para dor devido seus benefícios. Portando a cannabis medicinal apresentou efeitos significantes no tratamento da FM.

\section{Opioides}

Conforme Maffei ME (2020), opioides são muito utilizados no tratamento da FM ainda que não recomendados, já visto estudos clínicos sobre os efeitos negativos em pacientes se comparado a outros métodos terapêuticos. Alguns exemplos de opioides são a naltrexona, o tramadol, a codeína e o fentanil.

A naltrexona em baixas doses revelou-se eficaz e clinicamente benéfica no tratamento dos sintomas como a dor e fadigas diárias. Este opioide é considerado bem tolerado e seguro. Ainda, o tramadol, um agonista do receptor opioide sintético, proporciona certa alteração da dor, todavia, acompanhado de efeitos adversos como cefaleia, vertigem, náusea, constipação, vício, abstinência, sonolência e interações medicamentosas com antidepressivos. Outro opioide tido como fraco é a codeína. Pacientes com FM usuários da codeínaparacetamol relatam constipação e sonolência. Ademais, o fentanil, considerado 100 vezes mais potente que a morfina, atua inibindo a segunda onda de dor na FM, contudo, está muito associada a altos índices de overdoses e mortes relacionadas a sua utilização. Além da monoterapia, os usuários de opioides ainda podem associar o consumo de cafeína na terapêutica, podendo ser um potencial adjuvante opiáceo em pacientes de dor crônica (MAFFEI ME, 2020).

\section{Inibidores de recaptação de serotonina e norepinefrina (IRSNs)}

De acordo com Chinn S, et al. (2016) e em concordância com Maffei ME (2020), estudos feitos com a duloxetina e milnaciprano em $50 \%$ dos casos ou mais não houve melhora na qualidade de vida dos pacientes com FM, sendo a fadiga diária clinicamente irrelevante para essa terapia. A duloxetina demonstrou-se em vários ensaios com placebo conferir melhora na dor e nos sintomas depressivos. Já a venlafaxina permanece ainda escassos os estudos sobre sua eficácia em relação à dor crônica, todavia se revelou bem tolerada e de menor custo, sendo assim bem acessível ao tratamento da FM. Ademais, a Mirtazapina aparentou ser uma terapia promissora para melhora do sono, da dor e da qualidade de vida desses pacientes.

\section{Antidepressivos tricíclicos (ADTs)}

A amitriptilina como representante desta classe se mostrou bem-sucedida no tratamento da FM, sendo a terapêutica de primeira linha da FM. Estudos com esta revelaram sua eficácia na melhora da fadiga, da dor e também da qualidade de vida, tendo melhores efeitos que os IRSNs duloxetina e milnaciprano, entretanto a amitriptilina atinge $o$ alívio satisfatório da dor apenas pela minoria dos pacientes com FM (MAFFEI ME, 2020; CHINN S, et al., 2016).

\section{Drogas antiepilépticas (DAEs)}

Gabapentina e pregabalina são representantes desta classe, ambos atuam nos canais de íon cálcio dependente de voltagem no sistema nervoso central. Estudos com a pregabalina mostraram sua eficácia na redução da dor e nos distúrbios de sono e pouca relevância no tratamento da fadiga. Ainda, a pregabalina apresentou risco-benefício muito bom devido sua tolerabilidade em combinação com antidepressivos. Já a gabapentina ainda não existem evidências suficientes para comprovar sua ação na redução da dor na fibromialgia (MAFFEI ME, 2020; CHINN S, et al., 2016). 


\section{CONSIDERAÇÕES FINAIS}

A fibromialgia (FM) é uma síndrome crônica de etiologia incerta ligada a causas multifatoriais, que abrange sintomas inespecíficos, caracterizados por um quadro de dor musculoesquelética correlacionada a outras múltiplas manifestações clínicas. E, o tratamento dessa doença é indefinido, com baixa evidência científica, baseado em terapêuticas farmacológicas e não farmacológicas ainda sem resultados relevantes a curto e longo prazo para alívio da dor. Dentre as formas de intervenção propostas, destacam-se a prática de exercícios físicos aeróbicos, aquáticos e mistos. Além disso, o manejo pode incluir a neurodinâmica ativa e a estimulação transcraniana do córtex pré-frontal dorsolateral. Em suma, a FM é uma síndrome que carece de mais pesquisa e aprofundamento quanto a compreensão de sua propedêutica.

\section{REFERÊNCIAS}

1. ASSUMPÇÃO A, et al. Muscle stretching exercises and resistance training in fibromyalgia: which is better? A threearm randomized controlled trial. European Journal of Physical and Rehabilitation Medicine. 2018; 54(5): 663-670.

2. BENLIDAYI CI. Role of inflammation in the pathogenesis and treatment of fibromyalgia. Rheumatology International. 2019; 39(5): 781-791.

3. BIDONDE J, et al. Aerobic exercise training for adults with fibromyalgia. Cochrane Database of Systematic Reviews. 2017; 6(6): CD012700.

4. BIDONDE J, et al. Whole body vibration exercise training for fibromyalgia. Cochrane Database of Systematic Reviews. 2017;9(9): CD011755.

5. BIDONDE J, et al. Mixed exercise training for adults with fibromyalgia. Cochrane Database of Systematic Reviews. 2019; 5(5): CD013340.

6. CABO-MESEGUER A, et al. Fibromyalgia: Prevalence, epidemiologic profiles and economic costs. Medicina Clínica. 2017; 149(10): 441-448.

7. CECA D, et al. Benefits of a self-myofascial release program on health-related quality of life in people with fibromyalgia: A randomized controlled trial. Journal of Sports Medicine and Physical Fitness. 2017; 57(7-8): 993-1002.

8. CHINN S, et al. Fibromyalgia Pathogenesis and Treatment Options Update. Current Pain and Headache Reports. 2016; 20(4): 25.

9. CHOY EH. Current treatments to counter sleep dysfunction as a pathogenic stimulus of fibromyalgia. Pain management. 2016; 6(4): 339-46.

10. D'AGNELLI S, et al. Fibromyalgia: Genetics and epigenetics insights may provide the basis for the development of diagnostic biomarkers. Molecular Pain. 2019; 5: 1744806918819944.

11. DE LA VEGA R, et al. Fibroline: A mobile app for improving the quality of life of young people with fibromyalgia. Journal of Health Psychology. 2018; 23(1): 67-78.

12. DE LORENA SB, et al. Avaliação de dor e qualidade de vida de pacientes com fibromialgia. Revista Dor, 2016; 17(1): 8-11.

13. DI PIERRO F, et al. Role for a water-soluble form of CoQ10 in female subjects affected by fibromyalgia. A preliminary study. Clinical and Experimental Rheumatology. 2017; 105(3): 20-27.

14. ERICSSON A, et al. Resistance exercise improves physical fatigue in women with fibromyalgia: A randomized controlled trial. Arthritis Research and Therapy. 2016; 18: 176.

15. FITZGIBBON BM, et al. Evidence for the improvement of fatigue in fibromyalgia: A 4-week left dorsolateral prefrontal cortex repetitive transcranial magnetic stimulation randomized-controlled trial. European Journal of Pain. 2018; 22(7): 1255-1267.

16. HÄUSER W, et al. Fibromyalgia. Nature Reviews Disease Primers. 2015; 1: 15022.

17. HERMANS L, et al. Influence of Morphine and Naloxone on Pain Modulation in Rheumatoid Arthritis, Chronic Fatigue Syndrome/Fibromyalgia, and Controls: A Double-Blind, Randomized, Placebo-Controlled, Cross-Over Study. Pain Practice. 2017; 18(4): 418-430.

18. HEYMANN RE, et al. Novas diretrizes para o diagnóstico da fibromialgia. Revista Brasileira Reumatologia. 2017; $57(2)$ : 467-476.

19. KALLESE KH, et al. Comparing group-based acceptance and commitment therapy (ACT) with enhanced usual care for adolescents with functional somatic syndromes: A study protocol for a randomised trial. British Medical Journal Open. 2016; 6(9): e012743.

20. KIM SY, et al. Flexibility exercise training for adults with fibromyalgia. Cochrane Database of Systematic Reviews. 2019; 9(9): CD013419.

21. LITTLEJOHN G, GUYMER E. Neurogenic inflammation in fibromyalgia. Seminars in Immunopathology. 2018; 40(3): 291-300. 
22. MADDALI BONGI S, et al. Efficacy of rehabilitation with Tai Ji Quan in an Italian cohort of patients with Fibromyalgia Syndrome. Complementary Therapies in Clinical Practice. 2016; 24: 109-15.

23. MAFFEI ME. Fibromyalgia: Recent Advances in Diagnosis, Classification, Pharmacotherapy and Alternative Remedies. International Journal of Molecular Sciences. 2020; 21(21):787

24. MARTINEZ JE, et al. EpiFibro (Registro Brasileiro de Fibromialgia): dados sobre a classificação do ACR e preenchimento dos critérios diagnósticos preliminares e avaliação de seguimento. Revista Brasileira de Reumatologia. 2017; 57(2): 129-133.

25. MEEUS M, et al. Endogenous pain modulation in response to exercise in patients with rheumatoid arthritis, patients with chronic fatigue syndrome and comorbid fibromyalgia, and healthy controls: A double-blind randomized controlled trial. Pain Practice. 2015; 15(2): 98-106.

26. NOEHREN B, et al. Effect of transcutaneous electrical nerve stimulation on pain, function, and quality of life in fibromyalgia: A double-blind randomized clinical trial. Physical Therapy. 2015; 95(1): 129-40.

27. PRABHAKAR A, et al. The Role of Complementary and Alternative Medicine Treatments in Fibromyalgia: a Comprehensive Review. Current Rheumatology Reports. 2019; 21(5):14

28. REGLAND B, et al. Response to vitamin B12 and folic acid in myalgic encephalomyelitis and fibromyalgia. PLOS ONE. 2015; 10(4): e0124648.

29. RIVAS NEIRA S, et al. Effectiveness of Aquatic Therapy vs Land-based Therapy for Balance and Pain in Women with Fibromyalgia: A study protocol for a randomised controlled trial. BMC Musculoskeletal Disorders. 2017; 18(1): 22.

30. SARAL I, et al. The effects of long- and short-term interdisciplinary treatment approaches in women with fibromyalgia: a randomized controlled trial. Rheumatology International. 2016; 36(10):1379-1389.

31. SARZI-PUTTINI P, et al. Fibromyalgia: an update on clinical characteristics, aetiopathogenesis and treatment. Nature Reviews Rheumatology. 2020; 16(11): 645-660.

32. SILVA HJDA, et al. Mat Pilates and aquatic aerobic exercises for women with fibromyalgia: A protocol for a randomised controlled blind study. British Medical Journal Open. 2019; 9(2): e022306.

33. TO WT, et al. Differential effects of bifrontal and occipital nerve stimulation on pain and fatigue using transcranial direct current stimulation in fibromyalgia patients. Journal of Neural Transmission. 2017; 124(7): 799-808.

34. TORRES JR, et al. Results of an Active Neurodynamic Mobilization Program in Patients with Fibromyalgia Syndrome: A Randomized Controlled Trial. Archives of Physical Medicine and Rehabilitation. 2015; 96(10): 1771-1778.

35. UYSAL SC, et al. Effectiveness of the muscle energy technique on respiratory muscle strength and endurance in patients with fibromyalgia. Journal of Back and Musculoskeletal Rehabilitation. 2019; 32(3): 411-419.

36. WHITE HD, et al. Treatment of pain in fibromyalgia patients with testosterone gel: Pharmacokinetics and clinical response. International Immunopharmacology. 2015; 27(2): 249-256. 